\title{
Two-dimensional system based on organic and functional impairment with aging for precisely assessing risk factors in chronic disease
}

\author{
Wang Cengfang ${ }^{1}$, Gao Hanboya ${ }^{1}$, Liu Hui ${ }^{2}$
}

\author{
${ }^{1}$ Department of Heath Management, The First Affiliated Hospital of Dalian Medical \\ University, Dalian, China \\ 2Department of Clinical Immunology, College of Medical Laboratory, Dalian Medical \\ University, Dalian, China
}

Submitted: 22 March 2017

Accepted: 10 July 2017

Arch Med Sci 2020; 16 (1): 129-134

DOI: https://doi.org/10.5114/aoms.2020.91289

Copyright $\odot 2019$ Termedia \& Banach

\section{Abstract}

Introduction: The aim of the study was to establish a two-dimensional system based on organic and functional biological age (BA) using a biomarker of organic and functional impairment with aging for precisely assessing roles of natural aging and other risk factors in chronic disease.

Material and methods: The index of organic mild impairment, which is similar to the frailty index, was determined with imaging parameters as organic $B A$. The functional BA was calculated with the following formula: Functional $\mathrm{BA}=[$ systolic - diastolic BP $(\mathrm{mm} \mathrm{Hg})] \times$ waist circumference ${ }^{2}(\mathrm{~m}) \times$ height $^{0.5}$ $(\mathrm{m}) /$ weight $(\mathrm{kg})$. A total of 1047 subjects undergoing routine health examination were randomly selected to establish a system with organic BA on the $\mathrm{X}$-axis, functional BA on the $\mathrm{Y}$-axis; organic and functional BAs from chronological age with 60 years as a center point of the coordinate system. A total of 85 patients with type 2 diabetes controls were recruited to verify the system.

Results: Organic and functional BA contributed to chronological age almost equally. Both organic and functional BA increased with chronological age in the two-dimensional system for a random population. There were significant difference for distribution between diabetes and control groups in two-dimensional system $(p<0.05)$. That both more in quadrant II and lesser in quadrant IV for diabetes were suggested that diabetes was more sensitive to other risk factors than aging.

Conclusions: Combination of the two BAs could precisely recognized aging and other risk factors according to locations of the disease in each quadrant and quantitatively assessed status of nature aging and disease-related aging in a individual.

Key words: aging, biological age, obesity, blood pressure, biomarker.

\section{Introduction}

The incidence and development of chronic diseases are related to various factors, such as aging, genetics, smoking, overweight, and hyperlipidemia [1-4]; however, these risk factors are not necessarily associated with any particular disease. It is well known that incidence of chronic diseases increases with age, therefore, aging is believed to be the most significant risk factor for developing chronic diseases [5-7]. We believe that

\author{
Corresponding author: \\ Liu Hui \\ Department of Clinical \\ Immunology \\ College of \\ Medical Laboratory \\ Dalian Medical University \\ 116044 Dalian, China \\ Phone: +8641186110383 \\ E-mail: liuhui60@sina.com
}


reliability of these risk factors with lack of aging as an integer is questionable due to our previous study which found that the role of aging was over $80 \%$ in most chronic diseases [7]. It is necessary to evaluate the characteristics of chronic diseases using 2 properties: (1) aging and (2) other risk factors excluding aging.

Biological age (BA) is an objective expression of the degree of aging and can be determined using a variety of methods and models [8-10]. BA is typically determined from characteristics such as body shape [11-13], skin wrinkling [14-16], and graying of the hair [17-19], which are known to change with time. BA is also determined from physiological indexes, such as the physical frailty [20-22], blood pressure (BP) [23], heart rate [24], pulmonary function $[8,25]$, and from brain function variables, such as memory and arithmetic ability $[26,27]$, which are also known to change with age. We believe that these biomarkers can be classified as organic and functional impairment with aging and two types of BA can be defined using organic and functional biomarkers as functional BA and organic BA respectively.

Aging is also a typically complex process to which a hitherto unknown number of genes contribute by interacting with each other and the external factor [28-30]. Here, "external factor" refer to any cause that is not inherited genetically. The external factors that contribute to aging also contribute to chronic diseases [31]. Because the organic BA could be uncontrollable, the organic BA could be affected by genetic factors; whereas, compared with organic BA, much, but not all, functional BA can be controlled, the functional BA could relatively be affected by external factors in cluding environmental quality, quality of material life and quality of social life. Natural aging and other risk factors excluding aging could precisely be recognized with combination of the 2 BAs in chronic diseases. We found that these 2 BAs varied significantly in diabetes mellitus. A better understanding of 2 BAs' associations will be beneficial for developing a theoretical basis for strategies to prevent and control chronic diseases.

\section{Material and methods}

\section{Subjects}

The Institutional Ethics Committee of Dalian Medical University approved the study and waived the need for written informed consent from the participants due to the observational nature of the study.

Subjects scheduled for routine health examinations from the First Affiliated Hospital of Dalian Medical University, China, between October 2012 and November 2012 were selected to establish the two-dimensional system. The inclusion criteria were that results of height, weight, waist circumference (WC), blood pressure, imaging ultrasounds, chest radiography and electrocardiography should be recorded.

Patients with type 2 diabetes ( 56 men and 29 women, average age was $67.1 \pm 13.4$ years) were selected from the First Affiliated Hospital of Dalian Medical University, China, between October 2012 and November 2012, according to these diagnostic criteria: 1) glycated hemoglobin $A_{1 c} \geq 6.5 \%$; 2) fasting plasma glucose $\geq 7.0 \mathrm{mmol} / \mathrm{l}$; or 3) blood glucose at $2 \mathrm{~h}$ in the oral glucose tolerance test $\geq 11.1 \mathrm{mmol} / \mathrm{l}$ [32]. The exclusion criterion was type 1 diabetes. Age- and gender-matched diabetic patients were recruited as a control group from above individuals with normal fasting plasma glucose (3.89-6.11 $\mathrm{mmol} / \mathrm{l})$ using stratified random sampling.

\section{Functional BA}

The height and weight of each subject were measured with height- and weight-measuring scales with precisions of $0.1 \mathrm{~cm}$ and $0.1 \mathrm{~kg}$, respectively. WC was measured using a measuring tape at the umbilical level.

The waist circumference density index (WCDI) was calculated using the following formula as an aging biomarker [33]:

$$
\text { WCDI }=\frac{W}{P^{2} \times \sqrt{H}}
$$

where $W$ represents weight $(\mathrm{kg}), H$ represents height $(\mathrm{m})$ and $P$ represents waist circumference $(\mathrm{m})$. The smaller the WCDI, the older is the age.

$\mathrm{BP}$ was measured with a standard mercury sphygmomanometer. The difference between systolic and diastolic BP (pulse pressure) was considered as an aging biomarker [34, 35]. The larger the difference, the older is the age.

The functional BA was calculated with the following formula:

Functional $\mathrm{BA}=\frac{S-D}{\mathrm{WCDI}}=\frac{(S-D) \times P^{2} \times \sqrt{H}}{W}$ where $S$ represents systolic $B P(\mathrm{~mm} \mathrm{Hg}), D$ represents diastolic BP $(\mathrm{mm} \mathrm{Hg}), W$ represents weight $(\mathrm{kg})$, $H$ represents height $(\mathrm{m})$ and $P$ represents WC $(\mathrm{m})$.

\section{Organic BA}

Organic mild impairment (OMI) is defined as gradually occurring minor pathological impairment in the organs that is mainly attributed to endogenous factors. OMI, which is not characterized by specific clinical symptoms, can be detected by imaging. In this study, the index of OMI (IOMI) was calculated and used as a quantitative biomarker of organic BA. The IOMI is similar to the frailty index, which is a possibility linking age-related accumulation of health deficits [36-38]. 
Determining the IOMI involved 6 examinations for organic impairment: 1) hepatic, 2) renal, 3) thyroid, 4) prostate (men only) or pelvic (women only) imaging ultrasounds, 5) chest radiography, 6) resting electrocardiography.

Ultrasonography was conducted with a probe frequency that ranged from 3.5 to $5 \mathrm{MHz}$. The examined organs included the liver, kidney, prostate (males) and uterus and its appendages (females), and thyroid. Before the examinations, all subjects fasted for over $8 \mathrm{~h}$. The abdominal examinations were conducted with the subjects in a supine position and in the left and right lateral positions. The subjects drank 1-1.5 I of water within 1-2 $\mathrm{h}$ before the ultrasound examination of the bladder, prostate (males) and uterus and its appendages (females) to ensure that the bladder was filled. Chest radiography was of the anteroposterior and lateral views. Electrocardiography was performed using 12 standard leads in the supine position.

Various pathological changes or suspected pathological changes in the above imaging reports, except for skeletal abnormalities, were defined as organic lesions in this study. Subjects without any specific imaging findings, except for skeletal abnormalities, were considered "normal" in this study. The result was evaluated by two to three physicians with the same protocol.

The IOMI could be calculated simply as the ratio of the deficits present in a person to the total number of deficits considered in a given study setting [36]. A score of 0 represented normal results and a score of 1 denoted abnormal results. The sum of the scores of the above tests was used to assess organic BA.

For example, if the hepatic and renal ultrasounds were abnormal while the other tests were normal, the value of organic change was $1+1+$ $0+0+0+0=2$. Organic BA was defined as the percentage of organs with $\mathrm{OMI}$ and was calculated as follows [36]: Organic BA = (value of organic change/6) $\times 100 \%$.

\section{Statistical analysis}

In evaluating the contribution of organic and functional BAs to aging, age was applied as the dependent variable, while the organic and functional BAs were used as the independent variable. The degree of contribution to aging was measured with $R^{2}$. The analyses were performed with SPSS (SPSS Inc., Chicago, IL). P-values < 0.05 (twotailed) were considered significant.

\section{Two-dimensional system}

We plotted the scatter diagram to consider the effects of organic and functional BAs on aging to- gether. The organic BA was plotted on the X-axis; functional BA was plotted on the Y-axis; organic and functional BAs from chronological age with 60 years was considered as a center point to set up the coordinate system. We used the locations of the various groups in this coordinate system to sort them according to whether functional BA was higher or lower than the organic BA.

\section{Risk assessment for diabetes}

Organic and functional BAs were obtained from patients with type 2 diabetes and subjects in control groups. The locations of the various groups in the coordinate system were used to assess diabetes by characteristics of risk from organic and functional BA. The constituent ratio in each quadrant of distribution in the diabetes group was compared with that in the control population using the $\chi^{2}$ test. $P<0.05$ was considered to indicate a statistically significant difference (two-tailed test).

\section{Results}

A total of 1047 subjects (432 men and 615 women, average age was $47.3 \pm 15.8$ years) were selected to establish regression equation and the two-dimensional system. The original data for organic and functional BA in each age group are presented in Table I. The regression equation with chronological age as the dependent variable and functional or organic BA as the independent variable was respectively calculated as follows: Age $=0.441$ organic $\mathrm{BA}+32.487 ; R^{2}=0.467 ; p$-value $<0.001 ;$ Age $=43.711$ functional $B A+17.900$; $R^{2}=0.494 ; p$-value $<0.001$.

The center point was 62.38 for organic BA and 0.96 for functional BA when chronological age was 60 years. A two-dimensional system could be established with organic BA as the $\mathrm{X}$-axis; functional $B A$ as the $Y$-axis and $60,1.0$ as the center point as shown in Figure 1. There were different distribution of subjects between fasting plasma glucose $\geq 6.11 \mathrm{mmol} / /$ and that $<6.11 \mathrm{mmol} / /$ from same population $(p<0.05)$.

Distribution of chronological age in the two-dimensional system is presented in Table II. There

Table I. Contribution of functional or organic BA to chronological age for random population

\begin{tabular}{|c|c|c|}
\hline Age groups [years] & $\begin{array}{c}\text { Organic BA } \\
(\text { mean } \pm S D)\end{array}$ & $\begin{array}{c}\text { Functional BA } \\
\text { (mean } \pm S D)\end{array}$ \\
\hline$>60$ & $26.41 \pm 19.80$ & $0.58 \pm 0.14$ \\
\hline$\leq 60$ & $62.44 \pm 20.20$ & $1.03 \pm 0.28$ \\
\hline$R^{2}$ & 0.467 & 0.494 \\
\hline
\end{tabular}




\begin{tabular}{lc|c|}
\multicolumn{3}{c}{ Functional BA } \\
& & \\
Glucose $\geq 6.11$ & $16.9 \%$ & $25.4 \%$ \\
Glucose $<6.11$ & $2.5 \%$ & $4.1 \%$ \\
\cline { 2 - 3 } & III & IV \\
Glucose $\geq 6.11$ & $40.0 \%$ & $17.7 \%$ \\
Glucose $<6.11$ & $85.5 \%$ & $7.9 \%$ \\
& $\left(\chi^{2}=172.465, p<0.001\right)$
\end{tabular}

Figure 1. Different distribution of subjects with fasting plasma glucose $\geq 6.11 \mathrm{mmol} / \mathrm{l}$ and those with $<6.11 \mathrm{mmol} / \mathrm{l}$ from same population in two-dimensional system with $(60,1.0)$ as coordinate origin

is a significant difference for distribution between each quadrant in the two-dimensional system $(p<0.05)$.

A total of 85 patients with type 2 diabetes (56 men and 29 women, average age was $67.1 \pm 13.4$ years) and age- and gender-matched controls were selected. The locations of the diabetes and control groups are presented in Figure 2. There is a significant difference for distribution between diabetes and control groups in the two-dimensional system $(p<0.05)$.

\section{Discussion}

The organic BA was determined based on imaging parameters; therefore, it was a semi-quantitative reflection of pathological change with aging. The functional BA was determined based on BP and obesity parameters; therefore, it was a reflection of change with aging in the endocrine system and metabolism. In this study, organic and functional BA were associated with chronological age and contributed to chronological age almost equally; the role of the two variables was independent of age, indicating that the two-dimensional system can be established by measuring organic and functional BA, which is the main rationale for using the two-dimensional system to evaluate aging, health and disease risk. Our results revealed that both organic and functional BA increased

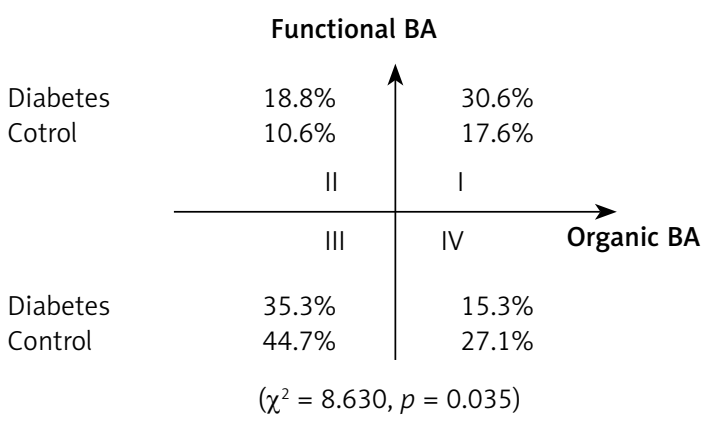

Figure 2. Locations of the diabetes and control groups with gender- and age-matched subjects in two-dimensional system with $(60,1.0)$ as coordinate origin

with chronological age in the two-dimensional system (Tables I and II), indicating that organic and functional BA as an integer were reasonable to assess the different effects of aging.

The original BA is relatively uncontrollable and mainly represents natural aging. The functional BA in this study is calculated with hypertension and obesity; the latter is also associated with many chronic diseases in both genetic and external factors [32, 39-41]; we considered functional BA as disease-associated aging for this reason. The risks for chronic diseases from aging and other factors could be discriminated through the two-dimensional system based on two BAs. Quadrant II in the two-dimensional system represents other risk factors that are not related to natural aging for a chronic disease. Quadrant IV represents risk from natural aging for a chronic disease. Quadrant I and quadrant III represent synergistic roles from both natural aging and other risk factors in a chronic disease.

It is well known that the incidence of diabetes mellitus increases greatly as a society develops and is more sensitive to external risk factors [42-45]. Therefore, diabetes mellitus was used as the reference disease in the present study. Subjects with higher fasting plasma glucose could be considered as a risk population for diabetes [44]; thus, subjects with fasting plasma glucose

Table II. Distribution of chronological age in two-dimensional system based on organic and functional BA for random population

\begin{tabular}{|c|c|c|c|c|}
\hline \multirow[t]{2}{*}{ Quadrant } & \multirow[t]{2}{*}{$N$} & \multirow[t]{2}{*}{ Age, mean \pm SD } & \multicolumn{2}{|c|}{ Quadrant I vs. Quadrant III } \\
\hline & & & $t$ & $P$-value \\
\hline I & 71 & $74.6 \pm 8.7$ & & \\
\hline II & 45 & $71.2 \pm 11.4$ & 23.658 & $<0.001$ \\
\hline III & 836 & $42.0 \pm 11.4$ & & \\
\hline IV & 95 & $62.4 \pm 14.1$ & & \\
\hline Total & 1047 & $47.3 \pm 15.8$ & - & - \\
\hline
\end{tabular}


$\geq 6.11 \mathrm{mmol} / \mathrm{l}$ and those with $<6.11 \mathrm{mmol} / \mathrm{l}$ from the same population were observed with the two-dimensional system (Figure 1). The frequencies in subjects with higher fasting plasma glucose were relatively higher than those in subjects with lower fasting plasma glucose within quadrant II and quadrant IV, suggesting that both natural aging and other risk factors played an important role in diabetes. The frequency in subjects with higher fasting plasma glucose was higher within quadrant I and it was lower within quadrant III, suggesting that there was an interaction between natural aging and other risk factors for development of diabetes. The difference of distribution between the diabetes and control groups with gender- and agematched subjects in the two-dimensional system was similar to that between subjects with higher and lower fasting glucose, except that the frequency of patients with diabetes within quadrant IV was lower than the control group with gender- and age-matched subjects (Figures 1 and 2). It may be that the role of natural aging in diabetes was eliminated using age-matched subjects with diabetes in the control group.

The two-dimensional system could also used to quantitatively assess the status of natural aging and disease-related aging according to locations in each quadrant for an individual. Quadrant I represents an individual under aging status and quadrant III implies an individual under non-aging status. Quadrant II suggests disease-related aging over natural aging; controllable risk factors could become a major risk for this individual in quadrant II; an emphasis on maintaining good living habits and increasing basal health status may contribute to disease prevention implying that primary prevention may be difficult. Quadrant IV suggests natural aging over disease-related aging; uncontrollable risk factors could become a major risk for this individual in quadrant IV.

Our findings also suggest that other factors excluding aging can easily be described with BP, waist circumference, height and weight, functional BA = [systolic - diastolic BP $(\mathrm{mm} \mathrm{Hg})] \times$ waist circumference $^{2}(\mathrm{~m}) \times$ height $^{0.5}(\mathrm{~m}) /$ weight $(\mathrm{kg})$; the value of functional BA should be compared with organic BA for an individual; functional $B A<1.0$ for 60 years was also suggested.

In conclusion, the two-dimensional system based on organic and functional BA could be a more sensitive predictor owing to the consensus having been obtained with earlier studies for diabetes $[39,40]$. It is obvious that understanding the abovementioned quantitative roles of aging and other risk factors in chronic disease will be beneficial for the primary prevention of chronic diseases. This feature may allow focused research on these other factors excluding aging to delay the functional BA process for identification of novel targets that can be used to create more effective and personalized chronic disease prevention strategies.

\section{Acknowledgments}

The study was supported by a grant from the National Natural Science Foundation of China (No. 30770724).

\section{Conflict of interest}

The authors declare no conflict of interest.

\section{References}

1. Dietz WH, Douglas CE, Brownson RC. Chronic disease prevention: tobacco avoidance, physical activity, and nutrition for a healthy start. JAMA 2016; 316: 1645-6.

2. Kimokoti RW, Millen BE. Nutrition for the prevention of chronic diseases. Med Clin North Am 2016; 100: 1185-98.

3. Hui L, Jing Y, Rui M, Weijian Y. Novel association analysis between 9 short tandem repeat loci polymorphisms and coronary heart disease based on a cross-validation design. Atherosclerosis 2011; 218: 151-5.

4. Hui L, Liping G, Jian Y, Laisui Y. A new design without control population for identification of gastric cancer-related allele combinations based on interaction of genes. Gene 2014; 540: 32-6.

5. The Lancet. Ageing in China: a ticking bomb. Lancet 2016; 388: 2058.

6. Hui L, Jun T, Jing Y, Yu W. Screening of cerebral infarction-related genetic markers using a Cox regression analysis between onset age and heterozygosity at randomly selected short tandem repeat loci. J Thromb Thrombolysis 2012; 33: 318-21.

7. Hui L. Aging and chronic disease as independent causative factors for death and a programmed onset for chronic disease. Arch Gerontol Geriatr 2015; 60: 178-82.

8. Belsky DW, Caspi A, Houts R, et al. Quantification of biological aging in young adults. Proc Natl Acad Sci USA 2015; 112: E4104-10.

9. Negasheva M, Lapshina N, Okushko R, Godina E. Biological age and tempos of aging in women over 60 in connection with their morphofunctional characteristics. J Physiol Anthropol 2014; 33: 12.

10. Levine ME. Modeling the rate of senescence: can estimated biological age predict mortality more accurately than chronological age? J Gerontol A Biol Sci Med Sci 2013; 68: 667-74.

11. Bae CY, Kang YG, Suh YS, Han JH, Kim SS, Shim KW. A model for estimating body shape biological age based on clinical parameters associated with body composition. Clin Interv Aging 2013; 8: 11-8.

12. Zhao X, Zhu S, Jia X, Yu L, Liu H. Constructing a waist circumference density index to predict biological age and evaluating the clinical significance of waist circumference density age. Exp Gerontol 2013; 48: 422-6.

13. Xin G, Shong L, Hui L. Effect of genetic and non-genetic factors, including aging, on waist circumference and $\mathrm{BMI}$, and inter-indicator differences in risk assessment. Exp Gerontol 2014; 60: 83-6.

14. Waaijer ME, Gunn DA, Catt SD, et al. Morphometric skin characteristics dependent on chronological and biolog- 
ical age: the Leiden Longevity Study. Age (Dordr) 2012; 34: 1543-52.

15. Purba MB, Kouris-Blazos A, Wattanapenpaiboon N, et al. Can skin wrinkling in a site that has received limited sun exposure be used as a marker of health status and biological age? Age Ageing 2001; 30: 227-34.

16. Waaijer ME, Parish WE, Strongitharm BH, et al. The number of p16INK4a positive cells in human skin reflects biological age. Aging Cell 2012; 11: 722-5.

17. Saeed M, Berlin RM, Cruz TD. Exploring the utility of genetic markers for predicting biological age. Leg Med (Tokyo) 2012; 14: 279-85.

18. Kocaman SA, Çetin M, Durakoğlugil ME, et al. The degree of premature hair graying as an independent risk marker for coronary artery disease: a predictor of biological age rather than chronological age. Anadolu Kardiyol Derg 2012; 12: 457-63.

19. Bulpitt CJ, Antikainen RL, Markowe HL, Shipley MJ. Mortality according to a prior assessment of biological age. Curr Aging Sci 2009; 2: 193-9.

20. Vidal El, Mayoral VF, Villas Boas PJ, Jacinto AF, Fukushima FB. Physical frailty as a clinical marker of biological age and aging. J Am Geriatr Soc 2015; 63: 837-8.

21. Cairney J, Veldhuizen S, Kwan M, Hay J, Faught BE. Biological age and sex-related declines in physical activity during adolescence. Med Sci Sports Exerc 2014; 46: 730-5.

22. Jee H, Jeon BH, Kim YH, et al. Development and application of biological age prediction models with physical fitness and physiological components in Korean adults. Gerontology 2012; 58: 344-53.

23. Melk A, Tegtbur U, Hilfiker-Kleiner D, et al. Improvement of biological age by physical activity. Int J Cardiol 2014 176: 1187-9.

24. Russoniello CV, Zhirnov YN, Pougatchev VI, Gribkov EN Heart rate variability and biological age: implications for health and gaming. Cyberpsychol Behav Soc Netw 2013; 16: 302-8.

25. Dekker P, van Baalen LM, Dirks RW, et al. Chronic inhibition of the respiratory chain in human fibroblast cultures: differential responses related to subject chronological and biological age. J Gerontol A Biol Sci Med Sci 2012; 67: 456-64.

26. Goggins WB, Woo J, Sham A, Ho SC. Frailty index as a measure of biological age in a Chinese population. J Gerontol A Biol Sci Med Sci 2005; 60: 1046-51.

27. Zhang WG, Zhu SY, Bai XJ, et al. Select aging biomarkers based on telomere length and chronological age to build a biological age equation. Age (Dordr) 2014; 36 : 9639.

28. An W, Zhang L, Gong B, Ren S, Liu H. Screening of longevity-associated genes based on a comparison between dead and surviving populations. Gene 2014; 534 379-82.

29. Liu H, Wang B, Liu D, et al. Molecular genetic studies on relationships among longevity, diseases, and HLADRB1/DQB1 allelic polymorphism. Exp Aging Res 2007; 33: $123-5$.

30. Hui L, Weijian Y, Xuelian D, Qigui L. Variations in short tandem repeats deduced on the basis of the number of repeats and the relationship of these variations with longevity. Age 2011; 33: 63-8.

31. Śliwczyński AM, Brzozowska M, Teter Z, Marczak M, Szymański P. Regional differences in the frequency of diabetes occurrence and its treatment costs during the years 2008-2013, based on the NFZ (National Health Fund) database. Arch Med Sci 2017; 13: 256-9.
32. Xin G, Shong L, Hui L. Effect of genetic and non-genetic factors, including aging, on waist circumference and $\mathrm{BMI}$, and inter-indicator differences in risk assessment. Exp Gerontol 2014; 60: 83-6.

33. Zhao X, Zhu S, Jia X, Yu L, Liu H. Constructing a waist circumference density index to predict biological age and evaluating the clinical significance of waist circumference density age. Exp Gerontol 2013; 48: 422-6.

34. Williams B. Vascular ageing and interventions: lessons and learnings. Ther Adv Cardiovasc Dis 2016; 10: 126-32.

35. AlGhatrif $M$, Wang M, Fedorova OV, Bagrov AY, Lakatta EG. The pressure of aging. Med Clin North Am 2017; 101: 81-101.

36. Wang C, Liu C, Gao H, Liu H. Order of aging of major human organs or systems and evaluation of health status based on aging. Biomarkers 2017; 22: 145-8.

37. Brothers TD, Theou O, Rockwood K. Do performancebased health measures reflect differences in frailty among immigrants age 50+ in Europe. Can Geriatr J 2014; 17: 103-7.

38. Peña FG, Theou O, Wallace L, et al. Comparison of alternate scoring of variables on the performance of the frailty index. BMC Geriatr 2014; 14: 25.

39. Ito $\mathrm{K}$, Iwane $\mathrm{M}$, Miyai $\mathrm{N}$, et al. Exaggerated exercise blood pressure response in middle-aged men as a predictor of future blood pressure: a 10-year follow-up. Clin Exp Hypertens 2016; 38: 696-700.

40. GBD 2015 Risk Factors Collaborators. Global, regional, and national comparative risk assessment of 79 behavioural, environmental and occupational, and metabolic risks or clusters of risks, 1990-2015: a systematic analysis for the Global Burden of Disease Study 2015. Lancet 2016; 388: 1659-724.

41. Weragoda J, Seneviratne R, Weerasinghe MC, Wijeyaratne SM. Risk factors of peripheral arterial disease a case control study in Sri Lanka. BMC Res Notes 2016 9: 508.

42. Hui L. Chronic diseases and societal development, based on the death-risk index. Epidemiology 2015; 26: e9-10.

43. Sherwin R, Jastreboff AM. Year in diabetes 2012: the diabetes tsunami. J Clin Endocrinol Metab 2012; 97: 4293-301.

44. Xin G, Yang G, Hui L. Study to assess whether waist circumference and changes in serum glucose and lipid profile are independent variables for the CETP gene. Diabetes Res Clin Pract 2014; 106: 95-100.

45. Śliwczyński AM, Brzozowska M, Teter Z, Marczak M, Szymański P. Regional differences in the frequency of diabetes occurrence and its treatment costs during the years 2008-2013, based on the NFZ (National Health Fund) database. Arch Med Sci 2017; 13: 256-9. 